\title{
FOUR INEQUALITIES OF VOLKMANN TYPE
}

\author{
WŁODZIMIERZ FECHNER
}

Abstract. We deal with four functional inequalities which are motivated by a result of A. ChaljubSimon and P. Volkmann from 1994 and by several later results concerning the following two equations:

$$
\begin{aligned}
\max \{f(x+y), f(x-y)\} & =f(x)+f(y) \quad(\text { for each } x, y) \\
\min \{f(x+y), f(x-y)\} & =|f(x)-f(y)| \quad(\text { for each } x, y) .
\end{aligned}
$$

The purpose of the paper is to establish some basic properties of the inequalities discussed and to compare them with some well known classical functional inequalities, such as the inequality of subadditivity or the inequality of Jensen-quasiconvexity. Mathematics subject classification (2010): 39B62.

Keywords and phrases: Functional inequality, Volkmann type inequalities, quasiconvexity, Jensenquasiconvexity.

\section{REFERENCES}

[1] J. ACZÉL, Sur les opérations définies pour nombres réels, Bull. Soc. Math. France 76 (1948), 59-64.

[2] J. ACZÉL, Lectures on functional equations and their applications, (Mathematics in Science and Engineering) New York and London: Academic Press. 19, 1966.

[3] K. BARON, P. VOLKMANn, Characterization of the absolute value of complex linear functionals by functional equations, semlv Seminar LV, No. 28, 10 pp. http://www.math.us.edu.pl/smdk

[4] A. Chaljub-Simon, P. Volkmann, Caractérisation du module d'une fonction additive a l'aide d'une équation fonctionnelle, Aequationes Math. 47 (1994), 60-68.

[5] R. CRAigen, Zs. PÁles, The associativity equation revisited, Aequationes Math. 37 (1989), 306312.

[6] W. FECHNER, Functional characterization of a sharpening of the triangle inequality, Math. Inequal. Appl. 13, 3 (2010), 571-578.

[7] R. GER, Fischer-Muszély additivity on Abelian groups, Comment. Math. Prace Mat. Tomus Specialis in honorem Iuliani Musielak (2004), 82-96.

[8] A. GilÁnyi, Volkmann type inequalities, talk at the 10th Debrecen-Katowice Winter Seminar on Functional Equations and Inequalities, Múvész Üdülő, Zamárdi (Hungary), February 3-6 2010.

[9] A. Gilányi, K. Nikodem, Zs. PÁles, Bernstein-Doetsch type results for quasiconvex functions, Math. Inequal. Appl. 7, 2 (2004), 169-175.

[10] H. J. Greenberg, W. P. Pierskalla, A review of quasiconvex functions, Operations Res. 19 (1971), 1553-1570.

[11] W. JARCZYK, P. VOLKMANN, On functional equations in connection with the absolute value of additive functions, Series Mathematicae Catoviciensis et Debreceniensis, No. 32, 11 pp. http://www.math.us.edu.pl/smdk

[12] T. KOCHANEK, On a composite functional equation fulfilled by modulus of an additive function, Aequationes Math. 80 (2010), 155-172.

[13] R. M. Redheffer, P. Volkmann, Die Funktionalgleichung $f(x)+\max \{f(y), f(-y)\}=$ $\max \{f(x+y), f(x-y)\}$, International Series of Numerical Mathematics 123, Birkhäuser Basel (1997), 311-318.

[14] A. W. Roberts, D. E. Varberg, Convex functions, Academic Press, New York-London, 1973. 